\title{
The effect of a warm electron beam on slow electron-acoustic solitons
}

\author{
L. N. Mbuli, ${ }^{1,2}$ R. Bharuthram, ${ }^{2}$ and S. K. Maharaj ${ }^{1,2}$ \\ ${ }^{1}$ South African National Space Agency (SANSA) Space Science, P.O. Box 32, Hermanus 7200, South Africa \\ ${ }^{2}$ University of the Western Cape, Robert Sobukwe Road, Bellville 7535, South Africa
}

(Received 29 August 2017; accepted 9 January 2018; published online 31 January 2018)

\begin{abstract}
The effects of the inclusion of finite drift speed of a warm electron component on the existence of arbitrary amplitude slow electron-acoustic solitons are investigated in a model with ions and cool, warm, and hot electrons. All plasma species are treated as adiabatic fluids. For fixed densities of the cool, warm, and hot electrons, the admissible Mach number ranges of the supported negative potential solitons are found to widen with increasing warm electron beam speed, up to a maximum value of $v_{d b w o}=0.7$. Beyond this maximum value, the soliton Mach number ranges become narrower and vanish completely at $v_{d b w o}=1.084$ where a switch to positive polarity solitons occurs. For a fixed value of the drift speed of the warm electrons, the cool electron density value at which the switch to positive polarity soliton occurs is the lowest when there is no streaming of the warm electrons but increases with increasing drift speed. Published by AIP Publishing.

https://doi.org/10.1063/1.5002095
\end{abstract}

\section{INTRODUCTION}

Broadband electrostatic noise (BEN) collectively describes electrostatic wave emissions in the broad frequency range of $10 \mathrm{~Hz}$ to $10 \mathrm{kHz}$. Electrostatic solitary waves (ESWs) which are the signatures of Broadband Electrostatic Noise have been observed by Geotail, ${ }^{1,2}$ Polar, ${ }^{3}$ and FAST $^{4}$ satellites in the plasma sheet boundary layer, magnetotail, and mid-altitude auroral regions of the Earth's magnetosphere, respectively. The bipolar pulses in the electric field which propagate parallel to the magnetic field have been observed in conjunction with electron flows which are directed parallel and/or antiparallel to the magnetic field direction. The short period duration of the bipolar pulses suggests that the ESWs are related to electron dynamics. The observations of the BEN waveforms have led to a number of theoretical and simulation studies which attempt to explain the formation of the ESWs. The theoretical interpretation of ESWs which form in the nonlinear stage of the electron beam instability is described in terms of a Bernstein-Green $\mathrm{Kruskal}^{5}$ (BGK) soliton which is a moving electrostatic potential perturbation (positive polarity) that is associated with a depletion in the electron density. The computer simulation studies of Omura et al. ${ }^{6}$ demonstrate the role of electron and ion beam instabilities in the generation of the ESWs. The simulations show that the temperature of the ions plays a much more significant role than the ion flow speed in the formation of the ESWs, which is in agreement with the characteristics of BEN inferred from satellite observations. ${ }^{2}$ Whilst kinetic studies $^{7-11}$ have shown that the linear electron-acoustic wave accounts for frequencies up to the local electron plasma frequency, solitons which form in the nonlinear stage of the electron-acoustic instability are likely to be responsible for frequencies which exceed the local electron plasma frequency. ${ }^{12,13}$ The mentioned studies highlight the importance of the contributions of both linear and nonlinear electron-acoustic waves to high frequency BEN.

The electron-acoustic mode with phase speed which lies between the cool and hot electron thermal speeds is a normal mode in a plasma with two-temperature electrons. On time scales associated with (high frequency) electron dynamics, the ions are immobile and provide neutrality in the (undisturbed) equilibrium state. The extension of the model to three-electron temperatures (cool, warm, and hot), naturally, invokes the presence of two electron-acoustic modes. The cool (warm) electrons provide the inertia, and the warm (hot) electrons provide the pressure in the propagation of the slow (fast) electron-acoustic modes. Detailed studies of arbitrary amplitude solitons associated with slow $^{14}$ and fast ${ }^{15}$ electron-acoustic modes have been conducted in models in which the cool, warm, and hot electrons are treated as adiabatic species.

The occurrence of slow-mode electron-acoustic solitons having negative potentials ${ }^{14}$ is limited in the order of increasing cool electron density by the cool and then the warm electron number densities becoming unreal and ultimately by the occurrence of double layers. A switch to positive potential solitons occurs at $n_{c e 0}=0.3$, and the positive potential solitons are limited by the occurrence of double layers. Kakad et al. ${ }^{16}$ considered a plasma model which is composed of two (cold) species of electrons (of which one species is a beam) and two-temperature ions. The ions in their model are considered to be very much hotter than the electrons, which justifies neglecting their inertia by treating them as Boltzmann distributed. In such a case, the coexistence of negative and positive potential solitons is shown.

Beams which are ubiquitous in space plasma environments provide a source for both linear instabilities and nonlinear structures. Berthomier et al. ${ }^{17}$ showed that the extension of a two-temperature electron model to 
include a warm beam of electrons favours the existence of positive polarity solitons. Subsequent studies by Cattaert et al., ${ }^{18}$ Maharaj et al., ${ }^{19}$ and Singh et al. ${ }^{20}$ in models which retain inertia of the hot ${ }^{18,19}\left(\mathrm{warm}^{20}\right)$ electrons have shown the existence of positive polarity solitons without treating the $\operatorname{hot}^{18,19}\left(\right.$ warm $\left.^{20}\right)$ electrons as drifting. The results of Sagdeev theory in predicting the existence of nonlinear structures have been directly applied to regions in the Earth's magnetosphere where counterstreaming electron beams are present. $^{21-23}$ Bipolar shaped pulses which appear in electric field data from the plasma sheet boundary layer ${ }^{21}$ and magnetosheath regions ${ }^{22}$ may be attributed to positive potential electron-acoustic solitons.

In this paper, we investigate the effects of incorporating finite drift speed of the warm electrons on slow electron-acoustic nonlinear structures. The modeling of the three-temperature electrons as adiabatic fluids in this and earlier ${ }^{14,15,21-23}$ studies is based on the assumption that the thermal speeds of the cool, warm, and hot electrons are not widely separated. Although the more commonly used and popular assumption is that the hot electrons are Boltzmann distributed (i.e., the hot electrons are effectively massless), when attempting to interpret satellite observations, ${ }^{1-4}$ for such models, electronacoustic type nonlinear structures do not yield positive ${ }^{24}$ polarity solitons, which have been measured in the plasma sheet boundary layer, ${ }^{1,2}$ magnetotail, ${ }^{3}$ and midaltitude auroral ${ }^{4}$ regions of the magnetosphere of Earth. The possibilities for refining the theoretical models such as retaining inertia of the warmer electrons and incorporating the effects of finite drift speed as considered in this study may better align the theoretical findings with observations. ${ }^{1-4}$

The plasma model and theory are presented in Sec. II. Numerical results and discussion are presented in Sec. III. The conclusions are presented in Sec. IV.

\section{PLASMA MODEL AND GOVERNING EQUATIONS}

In a four-component model with cool, warm, and hot electrons and ions (all are treated as adiabatic fluids), the dynamics of each species $j$ is governed by the equations of continuity, momentum, and pressure balance

$$
\begin{gathered}
\frac{\partial n_{j}}{\partial t}+\frac{\partial\left(v_{j} n_{j}\right)}{\partial x}=0 \\
\frac{\partial v_{j}}{\partial t}+v_{j} \frac{\partial v_{j}}{\partial x}=-\frac{Z_{j}}{\mu_{j}} \frac{\partial \Phi}{\partial x}-\frac{1}{\mu_{j} n_{j}} \frac{\partial P_{j}}{\partial x}, \\
\frac{\partial P_{j}}{\partial t}+v_{j} \frac{\partial P_{j}}{\partial x}+3 P_{j} \frac{\partial v_{j}}{\partial x}=0 .
\end{gathered}
$$

The set of equations is closed by Poisson's equation

$$
\frac{\partial^{2} \Phi}{\partial x^{2}}=n_{c e}+n_{h e}+n_{w e}-n_{i} .
$$

The above equations are presented in the normalised form. $Z_{j}=+1(-1)$ for ions (electrons), $n_{j}, v_{j}, P_{j}, \mu_{j}$ $=m_{j} / m_{e}$ denotes the number density, speed, pressure, and mass-ratio of species $j$, where $j=c e, w e, h e, i$ represents the cool electrons, warm electrons, hot electrons, and ions. The number densities are normalised by the total equilibrium plasma density $n_{0}=n_{c e 0}+n_{w e 0}+n_{h e 0}$. Time is normalised by the inverse of the total electron plasma frequency $\omega_{p e}^{-1}=\left(4 \pi n_{0} e^{2} / m_{e}\right)^{-1 / 2}$, speeds by the hot electron thermal speed $C_{h e}=\left(T_{h e} / m_{e}\right)^{1 / 2}$, spatial lengths by the Debye length $\lambda_{d}=\left(T_{h e} / 4 \pi n_{0} e^{2}\right)^{1 / 2}$, and potential by $T_{h e} / e$.

Transforming the above set of fluid equations to a frame which is co-moving with the nonlinear structure by considering $\zeta=x-M t$, where $M\left(=V / C_{h e}\right)$ is the normalised speed of the nonlinear structure, and using boundary conditions for localised solutions, $n_{j} \rightarrow n_{j 0}, P_{j} \rightarrow P_{j 0}=n_{j 0} T_{j}, v_{j} \rightarrow v_{d b j 0}$, $\Phi \rightarrow 0$ at $\zeta \rightarrow \pm \infty$, we obtain the expression for the density of species $j$

$$
\begin{aligned}
n_{j}= & \frac{n_{j 0}}{\sqrt{6 \delta_{j}}}\left\{\left(\left(M-v_{d b j 0}\right)^{2}+3 \delta_{j}-\frac{2 Z_{j} \Phi}{\mu_{j}}\right)\right. \\
& \left. \pm \sqrt{\left(\left(M-v_{d b j 0}\right)^{2}+3 \delta_{j}-\frac{2 Z_{j} \Phi}{\mu_{j}}\right)^{2}-12\left(M-v_{d b j 0}\right)^{2}}\right\},
\end{aligned}
$$

where $\delta_{j}=P_{j} /\left(\mu_{j} n_{j 0}\right)$, and in this generic form, all plasma species are allowed to drift with a speed $v_{d b j 0}$.

The expression (5) for the density is rewritten in the form proposed by Ghosh et $_{\mathrm{al}}{ }^{25}$

$$
\begin{aligned}
n_{j}= & {\left[\frac{n_{j 0}\left(\left(M-v_{d b j 0}\right)+\sqrt{3 \delta_{j}}\right)}{2 \sqrt{3 \delta_{j}}}\right] } \\
& \times \sqrt{1-\frac{2 Z_{j} \Phi}{\mu_{j}\left(\left(M-v_{d b j 0}\right)+\sqrt{3 \delta_{j}}\right)^{2}}} \\
& \pm \frac{n_{j 0}\left(\left(M-v_{d b j 0}\right)-\sqrt{3 \delta_{j}}\right)}{2 \sqrt{3 \delta_{j}}} \\
& \times \sqrt{\left.1-\frac{2 Z_{j} \Phi}{\mu_{j}\left(\left(M-v_{d b j 0}\right)-\sqrt{3 \delta_{j}}\right)^{2}}\right]} .
\end{aligned}
$$

The density expressions (6) are substituted in the transformed Poisson's equation, which, upon integration, yields the energy-integral like equation

$$
\frac{1}{2}\left(\frac{d \Phi}{d \zeta}\right)^{2}+V(\Phi, M)=0
$$

where the expression for the Sagdeev pseudo-potential is given by 


$$
\begin{aligned}
& V(\Phi, M)=\left[\frac{n_{w e 0}\left(\left(M-v_{d b w o}\right)+\sqrt{3 T_{w e}}\right)^{3}}{6 \sqrt{3 T_{w e}}}\left[1-\left(\sqrt{1+\frac{2 \Phi}{\left(\left(M-v_{d b w o}\right)+\sqrt{3 T_{w e}}\right)^{2}}}\right)^{3}\right]\right. \\
& \left.-\frac{n_{w e 0}\left(\left(M-v_{d b w o}\right)-\sqrt{3 T_{w e}}\right)^{3}}{6 \sqrt{3 T_{w e}}}\left[1-\left(\sqrt{1+\frac{2 \Phi}{\left(\left(M-v_{d b w o}\right)-\sqrt{3 T_{w e}}\right)^{2}}}\right)^{3}\right]\right] \\
& +\left[\frac{n_{c e 0}\left(M+\sqrt{3 T_{c e}}\right)^{3}}{6 \sqrt{3 T_{c e}}}\left[1-\left(\sqrt{1+\frac{2 \Phi}{\left(M+\sqrt{3 T_{c e}}\right)^{2}}}\right)^{3}\right]-\frac{n_{c e 0}\left(M-\sqrt{3 T_{c e}}\right)^{3}}{6 \sqrt{3 T_{c e}}}\left[1-\left(\sqrt{1+\frac{2 \Phi}{\left(M-\sqrt{3 T_{c e}}\right)^{2}}}\right)^{3}\right]\right] \\
& +\left[\frac{n_{h e 0}(M+\sqrt{3})^{3}}{6 \sqrt{3}}\left[1-\left(\sqrt{1+\frac{2 \Phi}{(M+\sqrt{3})^{2}}}\right)^{3}\right]-\frac{n_{h e 0}(M-\sqrt{3})^{3}}{6 \sqrt{3}}\left[1-\left(\sqrt{1+\frac{2 \Phi}{(M-\sqrt{3})^{2}}}\right)^{3}\right]\right] \\
& +\left[\frac{\mu_{i}\left(M+\sqrt{3 T_{i} / \mu_{i}}\right)^{3}}{6 \sqrt{3 T_{i} / \mu_{i}}}\left[1-\left(\sqrt{1-\frac{2 \Phi}{\mu_{i}\left(M+\sqrt{3 T_{i} / \mu_{i}}\right)^{2}}}\right)^{3}\right]\right. \\
& \left.-\frac{\mu_{i}\left(M-\sqrt{3 T_{i} / \mu_{i}}\right)^{3}}{6 \sqrt{3 T_{i} / \mu_{i}}}\left[1-\left(\sqrt{1-\frac{2 \Phi}{\mu_{i}\left(M-\sqrt{3 T_{i} / \mu_{i}}\right)^{2}}}\right)^{3}\right]\right] \text {, }
\end{aligned}
$$

with the restriction $v_{d b j 0}=0$ for the ions and cool and hot electrons $(j=i, c e, h e)$ and treating only the warm electrons as drifting $\left(v_{d b w 0} \neq 0\right)$. Only positive values for $v_{d b w 0}$ are used having assumed that the direction of the drift is aligned with the direction of wave propagation.

In the limit of small wave amplitudes, (8) can be Taylor expanded, yielding

$$
V(\Phi) \approx C_{2} \Phi^{2}+C_{3} \Phi^{3}+\cdots
$$

for $C_{2}=\frac{1}{2}\left(d^{2} V(\Phi) / d \Phi^{2}\right)_{\Phi=0}$ and $C_{3}=\frac{1}{6}\left(d^{3} V(\Phi) / d \Phi^{3}\right)_{\Phi=0}$, where the second derivative is given by

$$
\begin{aligned}
\left(\frac{d^{2} V(\Phi)}{d \Phi^{2}}\right)_{\Phi=0}= & \frac{n_{c e 0}}{M^{2}-3 T_{c e}}+\frac{n_{w e 0}}{\left(M-v_{b w 0}\right)^{2}-3 T_{w e}} \\
& +\frac{n_{h e 0}}{M^{2}-3}+\frac{1}{\mu_{i}\left(M^{2}-3\left(T_{c i} / \mu_{i}\right)\right)}
\end{aligned}
$$

and the third derivative is

$$
\begin{aligned}
\left(\frac{d^{3} V(\Phi)}{d \Phi^{3}}\right)_{\Phi=0}= & -\frac{3 n_{c e 0}\left(M^{2}+T_{c e}\right)}{\left(M^{2}-3 T_{c e}\right)^{3}}-\frac{3 n_{w e 0}\left(\left(M-v_{b w 0}\right)^{2}+T_{w e}\right)}{\left(\left(M-v_{b w 0}\right)^{2}-3 T_{w e}\right)^{3}} \\
& -\frac{3 n_{h e 0}\left(M^{2}+1\right)}{\left(M^{2}-3\right)^{3}}+\frac{3\left(M^{2}+\left(T_{c i} / \mu_{i}\right)\right)}{\mu_{i}^{2}\left(M^{2}-3\left(T_{c i} / \mu_{i}\right)\right)^{3}} .
\end{aligned}
$$

The small amplitude solution ${ }^{26}$ of (7) is given by

$$
\Phi=-\left(\frac{C_{2}}{C_{3}}\right) \operatorname{sech}^{2}\left(\sqrt{\left(-\frac{C_{2}}{4}\right) \zeta^{2}}\right) .
$$

The phase speeds of the linear modes are numerically obtained from $C_{2}=0$, with the lowest, intermediate, and highest $M$-values corresponding to the ion-acoustic, slow electron-acoustic, and fast electron-acoustic modes, respectively. The polarity of small-amplitude (Korteweg-de Vries) solitons governed by the solution (12) is consistent with the sign of (11).

The conditions governing the existence of solitons are $V(\Phi)=0, d V(\Phi) / d \Phi=0, d^{2} V(\Phi) / d \Phi^{2}<0$ at $\Phi=0$ and $V(\Phi)=0$ at $\Phi=\Phi_{0}$ with $V(\Phi)<0$ for $\Phi_{0}<\Phi<0$ $\left(0<\Phi<\Phi_{0}\right)$ for negative (positive) potential solitons. For double layers, the additional condition that has to be satisfied is $\left(d^{2} V(\Phi) / d \Phi^{2}<0\right)_{\Phi=\Phi_{0}}$, with $\Phi_{0}<0(>0)$ for negative (positive) potential double layers.

\section{NUMERICAL RESULTS AND DISCUSSION}

The admissible Mach number $M$ ranges for the existence of slow electron-acoustic solitons are shown as a function of drift speed of the warm electrons $v_{d b w o}$ in Fig. 1(a) for normalised cool electron density $n_{c e 0}=0.2$ and warm electron density $n_{w e}=0.3$. For a fixed value of $v_{d b w o}$, moving upwards in the direction of increasing $M$ in the figure, solitons are supported for a value of $M$ which is between the lower or critical $M$ value, $M_{\text {crit }}$, on the lower curve and the upper $M$ limit, $M_{\max }$, on the upper curve. The existence domain has been demarcated into five regions according to the reason for the upper $M$ limits. The corresponding maximum amplitudes of the solitons are shown in panel (b).

In Region I, negative polarity solitons occur which are limited by the occurrence of double layers, which is illustrated in Fig. 2 for a fixed value $v_{d b w o}=0.1$ for the warm electron drift speed. The polarity (negative) and the lower and upper soliton Mach number $M$ limits in the absence of 

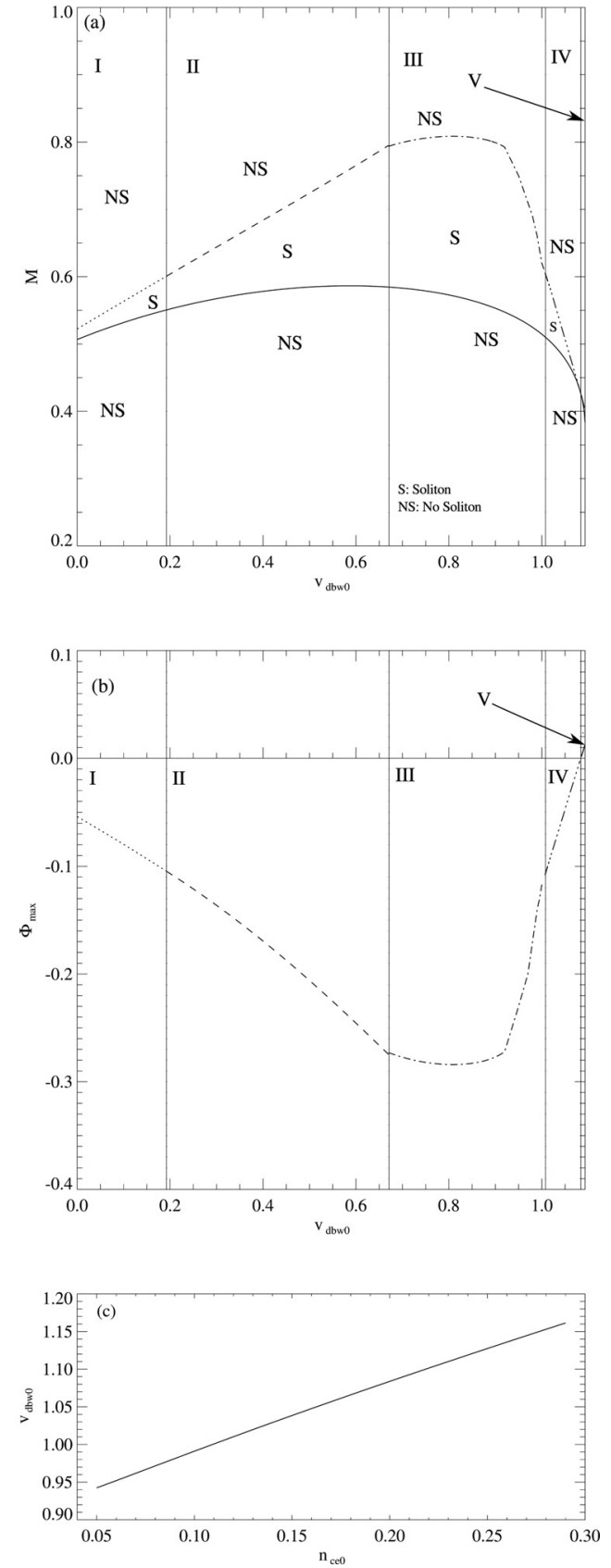

FIG. 1. (a) Existence domains for the slow electron-acoustic solitons as a function of the warm electron drift speed, $v_{d b w o}$, for a fixed value $n_{c e 0}=0.2$. (b) The corresponding maximum amplitudes for the slow electron-acoustic solitons are shown for a fixed value $n_{c e 0}=0.2$. The switch to positive polarity solitons is seen to occur at $v_{d b w 0}=1.084$ in panel (b). The panel (c) illustrates that the value $v_{d b w 0}$ corresponding to where the switch to positive polarity solitons occurs increases with increasing $n_{c e 0}$. The fixed input plasma parameters are $n_{w e 0}=0.3, \mu_{i}=1836, T_{c e}=T_{i}=0.001$, and $T_{w e}=0.25$.

streaming of the warm electrons $\left(v_{d b w o}=0\right)$ are consistent with the $M$-range displayed for $n_{c e 0}=0.2$ in Fig. 1 of the study by Mbuli et al. ${ }^{14}$ The lower limit $M=0.5319$ and the upper limit $M=0.5634$ lie on the lower and upper curves in Fig. 1(a), respectively. Figure 2 clearly shows that the upper $M$ limit coincides with the existence of a double layer. The polarity (negative) of the solitons and the occurrence of double layers as the upper limits on the soliton $M$ ranges corresponding to $v_{d b w 0}=0$ (Region I) in Figs. 1(a) and 1(b) are in

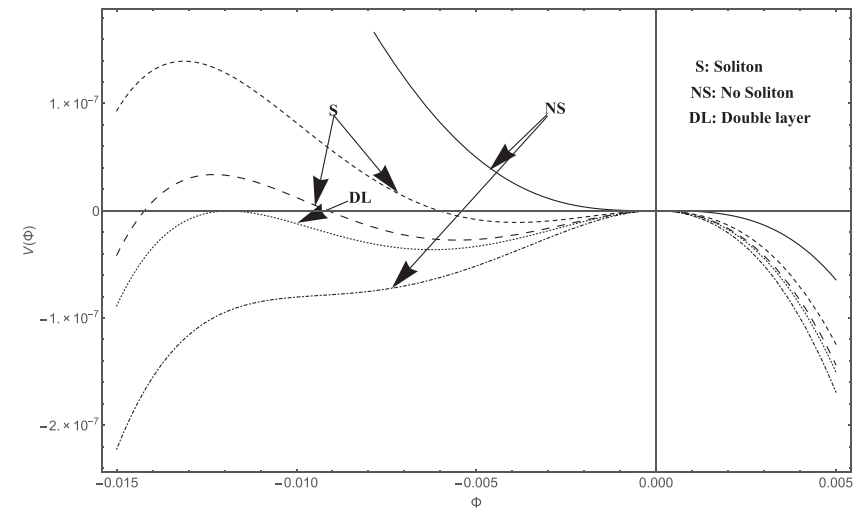

FIG. 2. Illustration of the Sagdeev pseudo-potential, $V(\Phi, M)$, profiles for the slow electron-acoustic solitons. The labelling parameter is the Mach number, $M=0.5319(-), M=0.55 \quad(--), M=0.56 \quad(---), M=0.5634$ $(\cdots)$, and $M=0.5655(-\cdot-\cdot-)$. The fixed input plasma parameters are those used in Fig. 1 (in Region I) with $v_{d b w o}=0.1$ and $n_{w e 0}=0.3$. The curve for $M=0.5634$ represents the double layer that limits the formation of solitons.

agreement with the results of Mbuli et al., ${ }^{14}$ for the cool and warm electron densities fixed at $n_{c e 0}=0.2$ and $n_{w e 0}=0.3$. A different combination of $n_{c e 0}$ and $n_{w e 0}$ would yield existence regions which differ from those shown in our Fig. 1 in view of the findings in Ref. 14 that the polarity of the supported solitons and the lower and upper limits on the soliton $M$ ranges are sensitive to a variation in the cool electron density $n_{c e 0}$ (for fixed $n_{w e 0}$ ) or the warm electron density $n_{w e 0}$ (for fixed $\left.n_{c e 0}\right)$.

For higher warm electron drift speeds, solitons in Region II of Fig. 1(a) are found to be limited by the warm electron density

$$
\begin{aligned}
n_{w e}= & \frac{n_{w e} 0}{2 \sqrt{3 T_{w e}}}\left(\sqrt{\left(\left(M-v_{d b w 0}\right)+\sqrt{3 T_{w e}}\right)^{2}+2 \Phi}\right. \\
& \left.+\sqrt{\left(\left(M-v_{d b w 0}\right)-\sqrt{3 T_{w e}}\right)^{2}+2 \Phi}\right)
\end{aligned}
$$

becoming unreal. This is illustrated in Fig. 3 for $v_{d b w 0}=0.65$, a value that corresponds to Region II.

Considering higher warm electron drift speeds, solitons in Region III are limited by the cool electron density

$$
\begin{aligned}
n_{c e}= & \frac{n_{c e} 0}{2 \sqrt{3 T_{c e}}}\left(\sqrt{\left(M+\sqrt{3 T_{c e}}\right)^{2}+2 \Phi}\right. \\
& \left.-\sqrt{\left(M-\sqrt{3 T_{c e}}\right)^{2}+2 \Phi}\right)
\end{aligned}
$$

becoming complex valued. The Sagdeev potentials in Fig. 4 look quite similar to those shown in Fig. 3; however, the solitons shown for $v_{d b w 0}=0.8$ in Fig. 4 are limited by the cool electron density (14) becoming unreal.

Solitons occurring for very high beam speeds $\left(v_{d b w 0}>1\right)$ in Region IV have negative potentials and are limited by double layers as confirmed by the occurrence of a double layer $(M=0.4491)$ for $v_{d b w o}=1.075$ in Fig. 5 .

Figure 1(a) demonstrates that the range of $M$-values for soliton formation increases with $v_{d b w 0}$ up to a maximum value of $v_{d b w o}=0.7$. Beyond this value, beam effects start to dominate the plasma and then there is a decrease in the $M$-number ranges until a switch to positive polarity solitons 


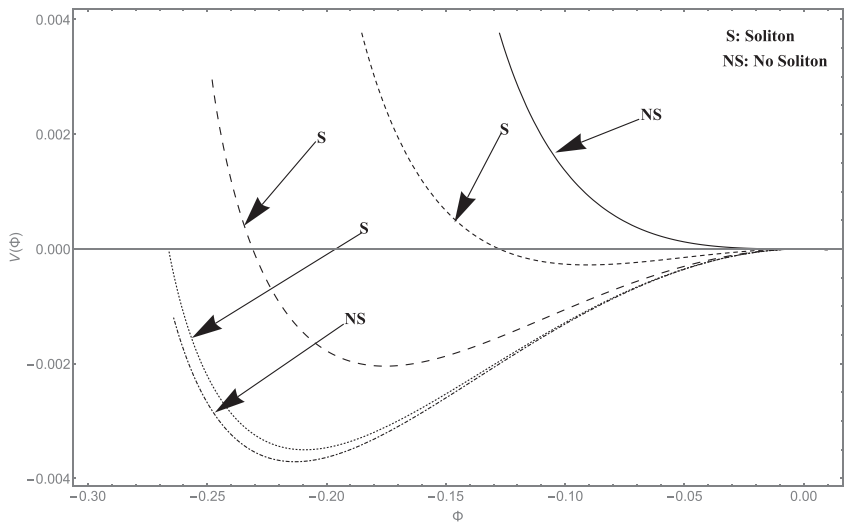

FIG. 3. Illustration of the Sagdeev pseudo-potential, $V(\Phi, M)$, profiles for the slow electron-acoustic solitons. The labelling parameter is the Mach number, $M=0.5854(-), M=0.6809(---), M=0.759(---), M$ $=0.7863(\cdots)$, and $M=0.7893(-\cdot-\cdot-)$. The fixed input plasma parameters are similar to those used in Fig. 1 (in Region II) with $v_{d b w o}=0.65$ and $n_{\text {we } 0}=0.3$. Solitons do not occur beyond $M=0.7863$ as the warm electron density $n_{w e}$ becomes unreal.

occurs at $v_{d b w o}=1.084$ where $M_{\text {crit }}=M_{\text {upper }}$ and $\Phi_{\max }=0$. Kinetic studies ${ }^{9,10}$ which are based on equivalent models show that with increasing speed of the beam of warm electrons, there is a transition from the electron-acoustic-instability to the beam-acoustic instability. The approximate solution for the frequency of the electron-acoustic mode which is coupled with a beam-acoustic mode as given in Ref. 9 is $\omega=k_{\|}\left[v_{b 0} \pm \frac{\omega_{b}}{k\left(1+1 /\left(k^{2} \lambda_{d h}^{2}\right)\right)^{1 / 2}}\right]$. The dominance of the electron-acoustic (beam) instability at low (high) values for the warm electron drift speed and the coupling between the two instabilities for moderate values of the beam speed as is

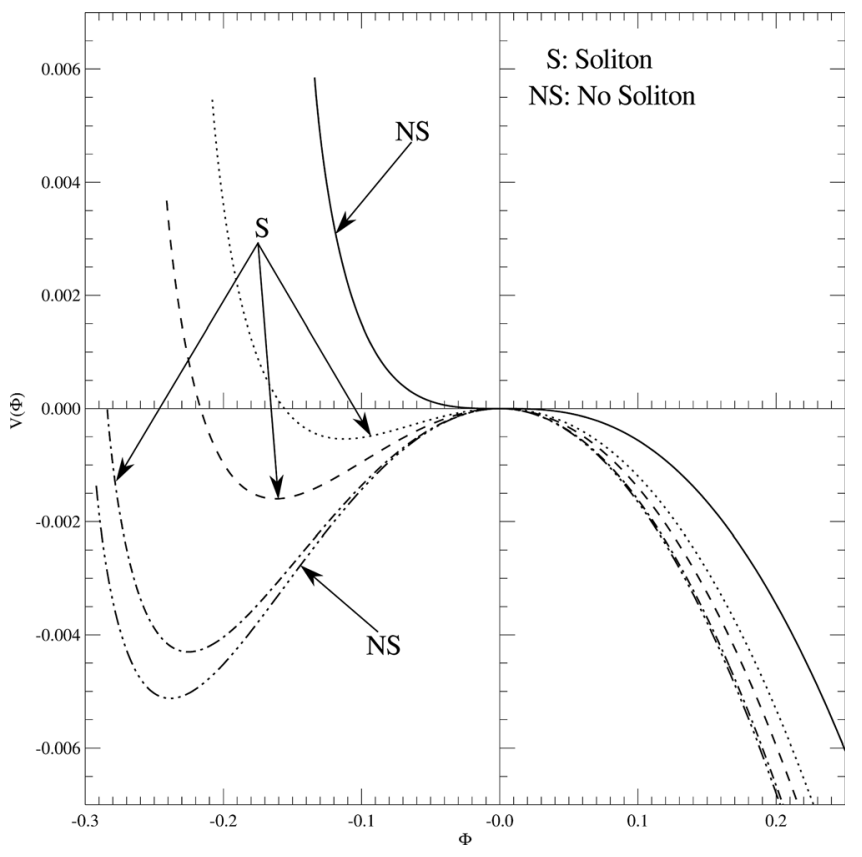

FIG. 4. Illustration of the Sagdeev pseudo-potential, $V(\Phi, M)$, profiles for the slow electron-acoustic solitons. The labelling parameter is the Mach number, $M=0.5729 \quad(-), M=0.7 \quad(\cdots), \quad M=0.75 \quad(--), \quad M$ $=0.8085(-\cdot-)$, and $M=0.82(-\cdots-)$. The fixed input plasma parameters are those used in Fig. 1 (in Region III) with $v_{d b w o}=0.8$ and $n_{w e 0}=0.3$. Solitons do not occur beyond $M=0.8085$ as the cool electron density $n_{c e}$ becomes unreal.

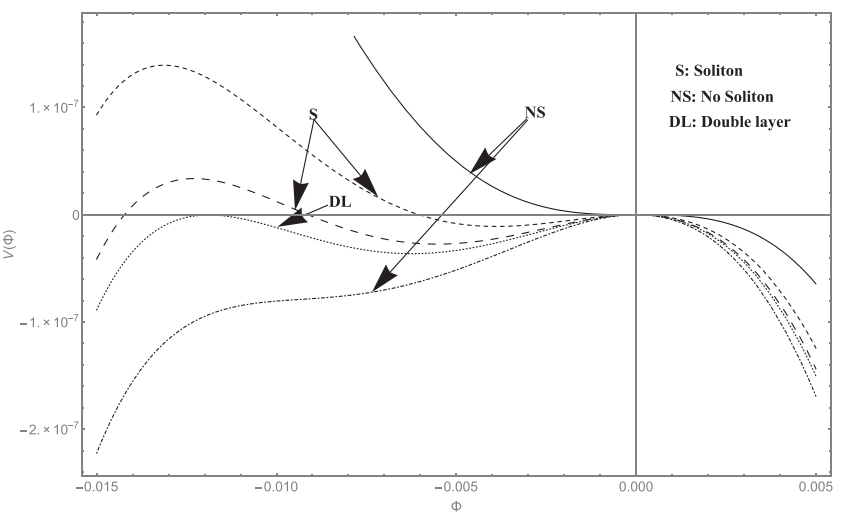

FIG. 5. Illustration of the Sagdeev pseudo-potential, $V(\Phi, M)$, profiles for the slow electron-acoustic solitons. The labelling parameter is the Mach number, $M=0.4451(-), M=0.4479(--), M=0.4488(---)$, $M=0.4491(\cdots)$ (double layer formed), and $M=0.45(-\cdot-\cdot-)$. The fixed input plasma parameters are those used in Fig. 1 (in Region IV) with $v_{d b w o}=1.075$ and $n_{w e 0}=0.3$.

apparent from the approximate expression (considering the lower signed solution) are very well aligned with numerical solutions for the real frequencies in Fig. 5 (Ref. 9) and Fig. 1 (Ref. 10). The reduction in both the lower $\left(M_{\text {crit }}\right)$ and upper ( $M_{\text {upper }}$ ) limits in the Mach number for the existence of slow electron-acoustic solitons which we observe in Fig. 1(a) for increasing large values of the beam speed (beyond $\left.v_{d b w 0}=0.7\right)$ indicates that there is a transition to the beamacoustic mode $\left(\omega / k=v_{d b w 0}\right)$, for which the associated nonlinear structures have only positive potentials. Consequently, the decrease in the lower and upper limits in $M$ for solitons in Regions III and IV is justified by the change in soliton polarity from negative to positive potentials (Region V) as a transition from the slow electron-acoustic to the beamacoustic mode occurs. Both the lower and upper $M$ limits for solitons decrease for increasing beam speeds beyond $v_{d b w 0}=0.7$. The ratio of the upper to lower $M$ limits for solitons, $M_{\text {upper }} / M_{\text {crit }}$, becomes smaller until ultimately a switch in soliton polarity occurs at the value $v_{d b w 0}=1.084$ where $M_{\text {upper }}=M_{\text {crit }}$ [Fig. 1(a)], $\Phi_{\max }=0$ [Fig. 1(b)], and the third derivative of the Sagdeev potential vanishes $\left.\left(d^{3} V(\Phi) / d \Phi^{3}\right)_{\Phi=0}=0\right)$ as the solitons switch polarity from negative $\left.\left(d^{3} V(\Phi) / d \Phi^{3}\right)_{\Phi=0}<0\right)$ potentials for $v_{d b w 0}<1.084$ to positive $\left.\left(d^{3} V(\Phi) / d \Phi^{3}\right)_{\Phi=0}>0\right)$ potentials for $v_{d b w 0}>1.084$. The value $v_{d b w 0}=1.084$ at which a switch to positive polarity solitons occurs is close to the minimum threshold value $v_{d b w 0}=1.094$ for which the slow electronacoustic mode becomes unstable. We recall that the sign of (11) gives the polarity of small amplitude solitons. The vanishing of the third derivative of the Sagdeev potential $\left.\left(d^{3} V(\Phi) / d \Phi^{3}\right)_{\Phi=0}=0\right)$ at $v_{d b w 0}=1.084$ and the reduction (increase) in the width of the soliton $M$ ranges for $v_{d b w 0}$ $<1.084\left(v_{d b w 0}>1.084\right)$ near $v_{d b w 0}=1.084$ are consistent with a very large number of studies ${ }^{14,15,18-20}$ in which polarity switches have been reported for electron-acoustic solitons. Positive polarity solitons occur for warm electron drift speeds $v_{d b w o}>1.084$ (Region V). The Sagdeev potentials shown in Fig. 6 for $v_{d b w o}=1.092$ confirm the existence of positive polarity solitons which are shown to be limited by 
the occurrence of a double layer $(M=0.4049)$. Considering that the maximum value of the warm electron drift speeds in our study is slightly more than that of the hot electron thermal speed, we conclude based on the results from kinetic theory, ${ }^{9,10}$ in particular, Fig. 5 (Ref. 9) and Fig. 1 (Ref. 10), that the range of warm electron drift speed values $\left(1.084<v_{d b w 0} \leq 1.093\right)$ in Figs. 1(a) and 1(b) where positive polarity solitons start to emerge is in the transition region between the electron-acoustic and beam-acoustic modes.

Figures 2-6 which show Sagdeev potentials for warm electron drift speed values typical of all five soliton regions are all similar in that we found only one polarity of soliton supported and not the coexistence of negative and positive potential solitons. We recall that in an earlier plasma model by Kakad et al. ${ }^{16}$ with two-temperature hot ion species which are treated as Boltzmann distributed, the coexistence of negative and positive potential solitons is shown to occur.

The existence regions as a function of $n_{c e 0}$ in Fig. 7 are shown for values of $v_{d b w o}$ fixed at 0 [panel (a)], 0.4 [panel (b)], and 0.9 [panel (c)]. In Fig. 7 [panels (a)-(c)], the progression of the upper $M$ limits for slow electron-acoustic solitons is imposed by the cool electron density (14) becoming unreal in Region I, the warm electron density (13) becoming unreal in Region II, negative polarity solitons limited by double layers in Region III, and positive polarity solitons which are limited by double layers in Region IV. Comparing with the expression (6), we point out that the negative sign is used in the density expression (14) for the cool electrons which are supersonic $\left(M>3 T_{c e}\right)$ for the slow electronacoustic mode, as opposed to the positive sign being used in the density expression (13) for the warm electrons which are subsonic $\left(M<3 T_{w e}\right)$ for the slow electron-acoustic mode. The switch to positive polarity solitons occurs at $n_{c e 0}=0.3$ [panel (a)], 0.475 [panel (b)], and 0.652 [panel (c)] for $v_{d b w o}$ $=0,0.4$, and 0.9 , respectively. The Mach number ranges for solitons increase in Regions I and II and decrease in Regions III and IV with an increase in warm electron drift speed. The general trend observed in Fig. 7(d) that the cool electron density at which the switch to positive polarity solitons occurs increases with the drift velocity is explained in terms of the

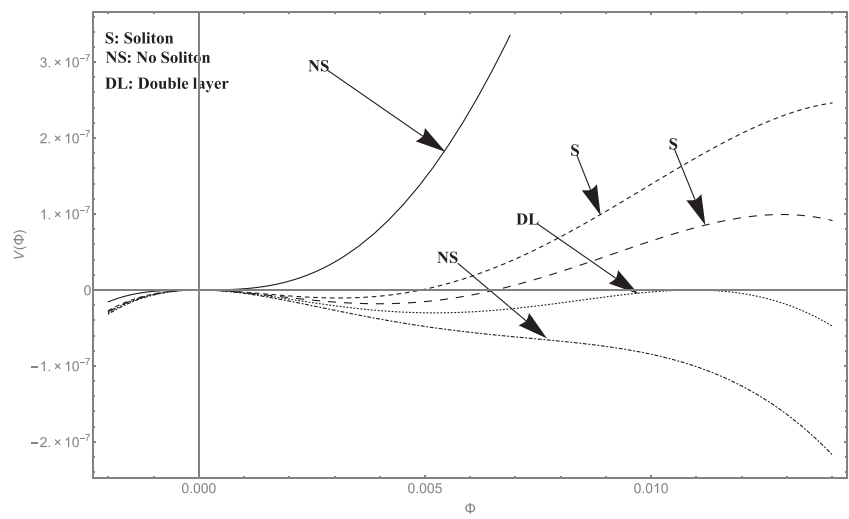

FIG. 6. Illustration of the Sagdeev pseudo-potential, $V(\Phi, M)$, profiles for the slow electron-acoustic solitons. The labelling parameter is the Mach number, $M=0.3939(-), M=0.403(---), M=0.404(---), M$ $=0.4049(\cdots)$ (double layer formed), and $M=0.406(-\cdot-\cdot-)$. The fixed input plasma parameters are those used in Fig. 1 (in Region V) with $v_{d b w o}=1.092$ and $n_{w e 0}=0.3$. large magnitude of the sum of the positive signed contributions of the warm and hot electrons in (11), which will require a large density of the cool electrons $n_{c e 0}$ [negative signed contribution to (11)] to reduce the (positive signed) contribution of the hot electrons, for balance. The (positive signed) contribution of the ions (fourth term) is negligible in that it can be ignored because of the large ion to electron mass ratio $\left(\mu_{i}=1836\right)$.

A model with counterstreaming ${ }^{23}$ electron beams and consideration of fast electron-acoustic solitons is beyond the scope of this paper; however, we will provide brief insights into the effects of counterstreaming beams on slow and fast electron-acoustic solitons. Our numerical computations for a model with a warm (speed $v_{1}$ ) and (counterstreaming) hot electron beam (speed $\left|v_{2}\right|$ ) having the same speed $\left(v_{1}=\left|v_{2}\right|=|v|\right)$ but different densities and temperatures (electron beams of the same speed, density, and temperature are considered in Ref. 23) reveal that the phase speed of the slow electron-acoustic mode increases with the beam speed of the counterstreaming beams (the ratio $M / M_{\text {crit }}$ decreases if $M$ is fixed). The phase speed of the fast electron-acoustic mode, on the other hand, decreases with increasing speed of the counterstreaming beams (the ratio $M / M_{\text {crit }}$ increases if $M$ is fixed). This explains the observed increase (decrease) in the negative (positive) potential slow (fast) soliton amplitudes with increasing beam speed in Ref. 23 as $M$ moves further away from (gets closer to) the value $M_{\text {crit }}$ at which solitons do not exist. We found that the ranges of beam speeds which support the existence of slow and fast electronacoustic solitons are very narrow; however, they are wider for the slow $(0 \leq|v| \leq 0.005)$ mode in comparison with the fast $(0 \leq|v| \leq 0.0002)$ mode which is in agreement with the results of Lakhina et al. ${ }^{23}$ For our choice of a low value of $n_{c e 0}=0.2$ for the cool electron density $\left(n_{c e 0}=0.5\right.$ was used in Ref. 23), we found that the slow and fast electron-acoustic solitons are limited by double layers. The negative potential slow electron-acoustic solitons in Ref. 23 which are found to be limited by one of the electron densities (as opposed to the double layer limit found in our study) are expected due to the different choice of value for the cool electron density. These findings will be presented in more detail in a forthcoming article.

\section{CONCLUSIONS}

In a model with cool, warm, and hot electrons and ions, two electron-acoustic modes are supported, if inertia is retained at least for the cool and intermediate temperature (warm) electrons. The slow electron-acoustic mode phase speed is between the thermal speeds of the cool and warm electrons $\sqrt{3} v_{t c e} \leq V(\equiv \omega / k) \leq \sqrt{3} v_{t w e}$. The fast electronacoustic mode phase speed lies between the warm and hot electron thermal speeds $\sqrt{3} v_{\text {twe }} \lesssim V(\equiv \omega / k) \lessgtr \sqrt{3} v_{\text {the }}$. The factor $\sqrt{3}$ arises from treating all species as adiabatic fluids and normalising the nonlinear structure speed with respect to a thermal speed (hot electrons) and neglecting the factor $\sqrt{3}$. The studies of slow ${ }^{14}$ and fast ${ }^{15}$ electron-acoustic solitons are based on the model composed of ions, cool, warm, and hot electrons in which all species are treated as adiabatic 

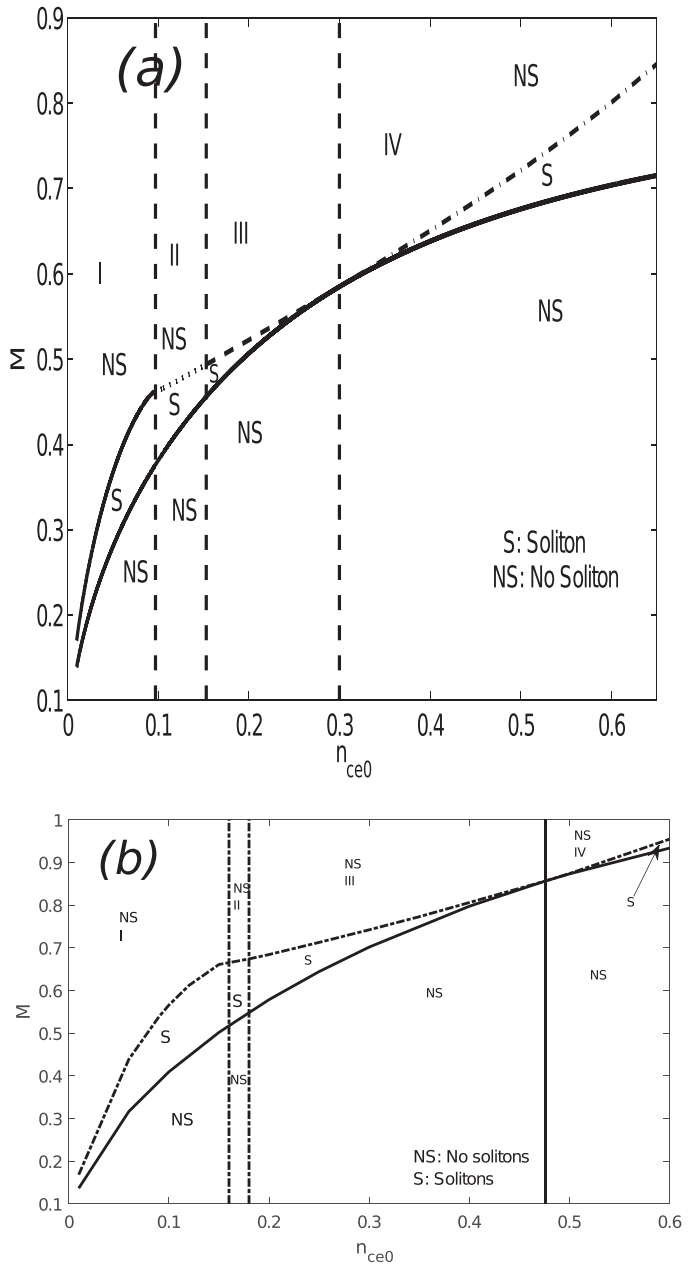
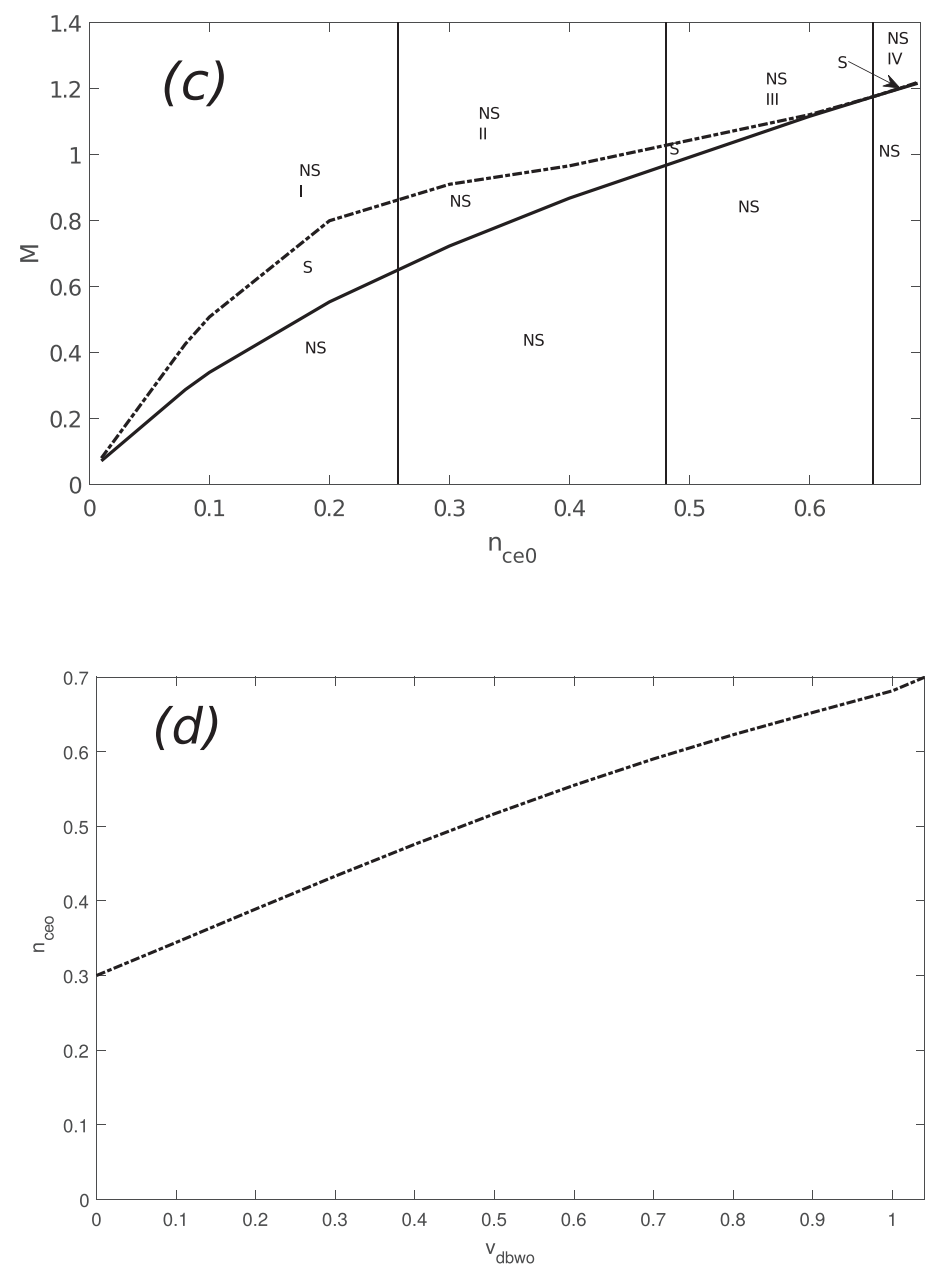

FIG. 7. In the upper left hand panel (a), we show the existence domain in the absence of an electron beam $\left(v_{d b w o}=0\right)$. It is noted that the polarity switch occurs at $n_{c e 0}=0.30$. In the remaining panels, we consider finite values of $v_{d b w o}$. In the lower left panel (b), for $v_{d b w o}=0.4 C_{h e}$, the polarity switch takes place at $n_{c e 0} / n_{o}=0.475$. In the upper right panel (c), the polarity switch is found at $n_{c e 0}=0.652 n_{o}$ for $v_{d b w o}=0.9$. In the lower right hand panel (d), the general behaviour of the $n_{c e 0} / n_{o}$ value for a polarity switch as a function of $v_{d b w o}$ is shown. The fixed input plasma parameters are those used in Fig. 1 with the warm electron number density fixed at $n_{w e 0}=0.3$.

fluids. The effects of incorporating finite drift speed for the warm electrons on the existence of slow electron-acoustic solitons have been investigated in this paper. The direction of the streaming of the warm electrons is assumed to be aligned with the direction of wave propagation.

Fixing the value of the cool $n_{c e 0}=0.2$ and warm $n_{w e 0}$ $=0.3$ electron densities, only negative potential solitons are supported for low values of the warm electron drift speed $v_{d b w o}$. The upper $M$ limits for negative potential solitons as $v_{d b w o}$ increases are imposed, respectively, by the occurrence of double layers, the warm and then the cool electron density becoming unreal, followed by solitons which are limited by the occurrence of (negative potential) double layers. The admissible soliton Mach number ranges are seen to widen with increasing drift speed of the warm electrons until an optimum value $v_{d b w o}=0.7$. Beyond $v_{d b w o}=0.7$, the width of the $M$-ranges starts to decrease until the value $v_{d b w o}=1.084$ is reached at which point $M_{\text {crit }}=M_{\text {upper }}$ and $\Phi_{\max }=0$, which signifies that a switch to positive polarity solitons occurs. At the switch-over point $\left(v_{d b w 0}=1.084\right)$, the soliton solution disappears $\left(\Phi_{\max }=0\right)$ and therefore the range of $M$ values $\triangle M=M_{\text {upper }}-M_{\text {crit }}=0$ as is seen in Fig. 1(a). The range of warm electron drift speeds $\left(1.084<v_{d b w 0} \leq 1.093\right)$, for which positive polarity solitons are supported, lies in the transition region between the slow electron-acoustic and beam-acoustic modes $\left(\omega / k=v_{d b w 0}\right)$ considering that the beam speed of the warm electrons is only marginally greater than the thermal speed of the hot electrons, ${ }^{9,10}$ and therefore, the solitons are associated with the coupled electron-acoustic and beam-acoustic modes. Considering the kinetic theory studies $^{9,10}$ which demonstrate that increasing beam speed of the warm electrons invokes a transition from an electronacoustic mode to a beam-acoustic mode, the positive polarity solitons are very likely associated with the slow electronacoustic mode which is coupled with a beam-acoustic mode.

The existence ranges as a function of the cool electron concentration for different fixed values of $v_{d b w o}$ demonstrate that the $n_{c e 0}$ value at which the switch to positive polarity solitons occurs increases with increasing drift speed of the warm electrons. The presented existence regions for slow electron-acoustic solitons show that the influence of including finite drift speed of the warm electrons has the effect of moving where the transition from negative to positive polarity solitons occurs in $v_{d b w o}\left(n_{c e 0}\right)$ with increasing $n_{c e 0}\left(v_{d b w o}\right)$. 
The range of $M$-values for soliton formation in the different existence regions is also dependent on the particular value of $v_{d b w o}$.

The importance of the role of drifting warm electrons in invoking a switch to positive potential solitons verified in this study is consistent with the theoretical findings of Berthomier et al. ${ }^{17}$ Furthermore, the consideration of warm electrons as a beam species in this study better aligns the theoretical model with plasmas which occur in the terrestrial magnetosphere as the association of high frequency broadband electrostatic noise with the presence of drifting electrons (beams) is very well established. ${ }^{1-4}$

${ }^{1}$ H. Matsumoto, H. Kojima, T. Miyatake, Y. Omura, M. Okada, I. Nagano, and M. Tsutsui, Geophys. Res. Lett. 21, 2915, https://doi.org/10.1029/ 94GL01284 (1994).

${ }^{2}$ H. Kojima, H. Matsumoto, T. Miyatake, I. Nagano, A. Fujita, L. A. Frank, T. Mukai, W. R. Paterson, Y. Saito, S. Machida, and R. R. Anderson, Geophys. Res. Lett. 21, 2919, https://doi.org/10.1029/94GL02111 (1994).

${ }^{3}$ J. R. Franz, P. M. Kintner, and J. S. Pickett, Geophys. Res. Lett. 25, 1277, https://doi.org/10.1029/98GL50870 (1998).

${ }^{4}$ R. E. Ergun, C. W. Carlson, J. P. McFadden, F. S. Mozer, G. T. Delory, W. Peria, C. C. Chaston, M. Temerin, R. Elphic, R. Strangeway, R. Pfaff, C. A. Cattell, D. Klumpar, E. Shelley, W. Peterson, E. Moebius, and L. Kistler, Geophys. Res. Lett. 25, 2025-2028, https://doi.org/10.1029/ 98GL00635 (1998).

${ }^{5}$ V. L. Krasovsky, H. Matsumoto, and Y. Omura, J. Geophys. Res. 102(A10), 22131-22139, https://doi.org/10.1029/97JA02033 (1997).

${ }^{6}$ Y. Omura, H. Matsumoto, T. Miyake, and H. Kojima, J. Geophys. Res. 101(A2), 2685-2697, https://doi.org/10.1029/95JA03145 (1996).

${ }^{7}$ D. Schriver and M. Ashour-Abdalla, J. Geophys. Res. 92(A6), 5807-5819, https://doi.org/10.1029/JA092iA06p05807 (1987).
${ }^{8}$ S. V. Singh and G. S. Lakhina, Planet. Space Sci. 49, 107-114 (2001).

${ }^{9}$ A. Sooklal and R. L. Mace, Phys. Plasmas 11, 1996 (2004).

${ }^{10}$ L. N. Mbuli, S. K. Maharaj, and R. Bharuthram, Phys. Plasmas 20(12), 122115 (2013).

${ }^{11}$ L. N. Mbuli, S. K. Maharaj, and R. Bharuthram, Phys. Plasmas 21(5), 052115 (2014).

${ }^{12}$ N. Dubouloz, R. Pottelette, M. Malingre, and R. A. Treumann, Geophys. Res. Lett. 18, 155-158, https://doi.org/10.1029/90GL02677 (1991).

${ }^{13}$ N. Dubouloz, R. A. Treumann, R. Pottelette, and M. Malingre, J. Geophys. Res. 98, 17415-17422, https://doi.org/10.1029/93JA01611 (1993).

${ }^{14}$ L. N. Mbuli, S. K. Maharaj, R. Bharuthram, S. V. Singh, and G. S. Lakhina, Phys. Plasmas 22(6), 062307 (2015).

${ }^{15}$ L. N. Mbuli, S. K. Maharaj, R. Bharuthram, S. V. Singh, and G. S. Lakhina, Phys. Plasmas 23(6), 062302 (2016).

${ }^{16}$ A. P. Kakad, S. V. Singh, R. V. Reddy, G. S. Lakhina, S. G. Tagare, and F. Verheest, Phys. Plasmas 14, 052305 (2007).

${ }^{17}$ M. Berthomier, R. Pottelette, M. Malingre, and Y. Khotyaintsev, Phys. Plasmas 7, 2987 (2000).

${ }^{18}$ T. Cattaert, F. Verheest, and M. A. Hellberg, Phys. Plasmas 12, 042901 (2005).

${ }^{19}$ S. K. Maharaj, R. Bharuthram, S. V. Singh, and G. S. Lakhina, Phys. Plasmas 19(12), 122301 (2012).

${ }^{20}$ S. V. Singh, G. S. Lakhina, R. Bharuthram, and S. R. Pillay, Phys. Plasmas 18, 122306 (2011).

${ }^{21}$ G. S. Lakhina, S. V. Singh, A. P. Kakad, and J. S. Pickett, J. Geophys. Res. 116, A10218, https://doi.org/10.1029/2011JA016700 (2011).

${ }^{22}$ G. S. Lakhina, S. V. Singh, A. P. Kakad, M. L. Goldstein, A. F. Vinas, and J. S. Pickett, J. Geophys. Res. 114, A09212, https://doi.org/10.1029/ 2009JA014306 (2009).

${ }^{23}$ G. S. Lakhina, S. V. Singh, and A. P. Kakad, Adv. Space Res. 47, 1558-1567 (2011).

${ }^{24}$ R. L. Mace, S. Baboolal, R. Bharuthram, and M. A. Hellberg, J. Plasma Phys. 45, 323-338 (1991).

${ }^{25}$ S. S. Ghosh, K. K. Ghosh, and A. N. Sekar Iyengar, Phys. Plasmas 3(11), 3939-3946 (1996).

${ }^{26}$ A. A. Mamun, Phys. Rev. E 77, 026406 (2008). 